\title{
POLÍTICA Y RELACIONES INTERNACIONALES
}

\section{AMÉRICA LATINA EN LA ZONA DE LA TURBULENCIA POLÍTICA}

\author{
Olga G. Leónova \\ Doctora titular (Politología),prof. (politolga@gmail.com) \\ Facultad de Estudios Globales \\ Universidad Estatal LOMONÓSOV de Moscú \\ Leninskie gory, 1, Moscú, 119991, Federación de Rusia \\ Recibido el 15 de diciembre de 2020 \\ Aceptado el 10 de febrero de 2021
}

DOI: $10.37656 / \mathrm{s} 20768400-2021-2-06$

Resumen. El mundo experimenta hoy la llegada de una etapa denominada la "nueva ola" de la globalización. Sus tendencias dieron origen a la crisis política global que se extendió también a los países de América Latina. Sin embargo, su manifestación en el continente tiene sus particularidades.

Los sintomas principales de la crisis en el continente son: la grave situación económica, la desaceleración del crecimiento económico, el déficit fiscal, la congelación de salarios, las consecuencias de la pandemia del coronavirus. En la esfera política: la debilidad institucional de los sistemas políticos y de los regímenes de los países de América Latina, que se expresa en violación de la legitimidad del poder estatal, en el ejercicio selectivo de la ley, en la corrupción del poder público, falta de control de la sociedad civil sobre el gobierno, la disminución del apoyo a la democracia, la politización de una serie de problemas económicos y sociales. En la esfera social: el ahondamiento de la desigualdad social, el incremento de la violencia y el narcotráfico, crisis migratoria.

La crisis se manifiesta, en primer lugar, en el final del "giro a la izquierda y en la tendencia al inicio de la "deriva a la derecha"; en segundo lugar, en el crecimiento de la actividad política de la población y de los movimientos de protesta.

La crisis política puede desembocar en un colapso de la democracia y en el reforzamiento de los rasgos autoritarios del sistema político en varios países de América Latina. Los hechos posteriores pueden generar la tendencia de la transformación de sus regímenes políticos y de la aplicación 
de la represión como medio de resolver la situación ante la ineficacia de las medidas económicas.

Palabras clave: América Latina, crisis, "nueva ola" de la globalización, giro a la derecha, giro a la izquierda, democracia, autoritarismo

\title{
LATIN AMERICA IN A ZONE OF POLITICAL TURBULENCE
}

\author{
Olga G. Leonova \\ Dr.Sci. (Politology), prof. (politolga@gmail.com) \\ Faculty of Global Studies \\ LOMONOSOV Moscow State University \\ 1, Leninskie gory, Moscow, 119991, Russian Federation \\ Received on December 15, 2021 \\ Accepted on February 10, 2021
}

DOI: $10.37656 / \mathrm{s} 20768400-2021-2-06$

Abstract. The global world today is experiencing a new stage, which is called the "New Wave" of globalization. New trends have created a global political crisis that spread to Latin America. However, its manifestation on the continent has its own specifics.

The main symptoms of the crisis on the continent are: a difficult economic situation, slowing economic growth, budget deficits, wage freezes, the consequences of the coronavirus pandemic. In the political sphere, there is institutional weakness of political systems and regimes in Latin America, which manifests itself in violation of the legitimacy of state power; the selective operation of the law; corruption of state power; restrictions on citizens ' access to security systems; lack of control of the government by civil society; reduced support for democracy and the politicization of a number of economic and social problems. In the social sphere, there is the deepening of social inequality, an increase in violence, drug trafficking, and the migration crisis.

The crisis manifests itself, firstly, in the end of the "left turn" ("Pink Tide") and trends towards the beginning of the "right drift"; secondly, in the growth of political activity and protest actions of the population.

The result of the political crisis may be the collapse of democracy and the strengthening of authoritarian features of the political system in some countries in Latin America. Further developments may give rise to a trend of 
transformation of their political regimes and the use of political repression as means of resolving the situation if economic measures prove ineffective.

Keywords: Latin America, the crisis, the globalization of the "new wave", a right turn, left turn, democracy, authoritarianism

\title{
ЛАТИНСКАЯ АМЕРИКА В ЗОНЕ ПОЛИТИЧЕСКОЙ ТУРБУЛЕНТНОСТИ
}

\author{
Ольга Георгиевна Леонова \\ Д-р полит.наук, проф., (politolga@gmail.com) \\ Факультет глобальных процессов \\ МГУ им. М.В. Ломоносова \\ РФ, 119991, Москва, Ленинские горы, 1 \\ Статья получена 15 декабря 2020 г. \\ Статья принята 10 февраля 2021 г.
}

DOI: $10.37656 / \mathrm{s} 20768400-2021-2-06$

Аннотация. Глобальный мир сегодня переживает наступление нового этапа, который назвали глобализацией «новой волны». Её новые тренды породили глобальный политический кризис, который распространился и на страны Латинской Америки. Однако в его проявлении на континенте есть своя специфика.

Основными симптомами кризиса на континенте являются: тяжелая ситуация в экономике, замедление темпов экономического роста, бюджетный дефиџит, замораживание зарплаты, последствия пандемии коронавируса. В политической сфере: институциональная слабость политических систем и режимов стран Латинской Америки, которая проявляется в нарушении легитимности государственной власти, избирательного действия закона, коррупции государственной власти, неподконтрольности власти гражданскому обществу, сокращении поддержки демократии, политизации ряда экономических и соииальных проблем. В сочиальной сфере: углубление сочиального неравенства, рост насилия, наркоторговли, миграционный кризис. Кризис проявляется, во-первых, в окончании «левого поворота» ('Pink Tide') и тенденции к началу «правого дрейфа»; во-вторых, в росте политической активности и протестных выступлений населения.

Следствием политического кризиса может быть сворачивание демократии и усиление авторитарных черт политической системы в ряде стран Латинской Америки. Дальнейшие события могут породить 
тенденцию трансформачии их политических режимов и применения политических репрессий как средство урегулирования ситуации в случае неэффективности экономических мер.

Ключевые слова: Латинская Америка, кризис, глобализаџия «новой волныl», правый поворот, левый поворот, демократия, авторитаризм

\section{“La nueva ola" de la globalización}

El análisis del desarrollo de los procesos de la globalización en la segunda década del siglo XXI revela que a lo largo del proceso del tránsito de la unipolaridad a la multipolaridad se manifiestan sus características nuevas.

Investigadores y expertos han comenzado a hablar hoy día de un "cambio de épocas" entendiendo por ello el advenimiento de una nueva etapa que bautizaron ya como "nueva ola" de la globalización [1]. Dicha etapa tiene una serie de rasgos nuevos. Entre ellos: la ampliación de los límites del mundo multipolar; el fortalecimiento de la interconexión e interdependencia de los sistemas local (regional) y global, a consecuencia de lo cual los problemas nacionales locales, si no se resuelven, pueden conducir a consecuencias catastróficas globales; más allá se caracteriza por la desmembración, la destrucción, la desintegración de algunos espacios geopolíticos, la turbulencia y la inestabilidad del mundo global.

Existen unas tendencias, comunes para todos los países del mundo global, que determinan el "rostro" de "la nueva ola" de la globalización. Éstas son: el crecimiento del proteccionismo en el comercio mundial; la desigualdad creciente de los ingresos y la exclusión social; el aumento del desempleo y del malestar social; el déficit fiscal de los países que participan activamente en los procesos económicos globales; la popularidad de la idea del nacionalismo "responsable"; la erosión de la democracia; la 
falta de seguridad a nivel nacional y global; el cambio climático y la migración forzosa.

Estos rasgos y tendencias típicas de la "nueva ola de la globalización" dieron origen a la crisis política mundial que se extendió también a los países de América Latina. Esta crisis desató airadas protestas y manifestaciones en vastos espacios del continente: desde Haití y Honduras hasta Bolivia, Ecuador, Chile y Venezuela. Sin embargo, su manifestación en el continente reveló particularidades propias.

Una de las más importantes razones que incentivó las protestas en el continente y que engendró síntomas de crisis política fue la grave situación económica de la mayoría de los países latinoamericanos.

América Latina, después del período de un enérgico crecimiento económico en la primera década del siglo XXI, entró en una fase de estancamiento, produciéndose en algunos países caídas de los indicadores económicos. La congelación de salarios, el alza del costo de la vida, los escándalos de corrupción, el agravamiento de la desigualdad social originaron una frustración cada vez mayor en la gente.

Una situación económica tan dura en la mayoría de los países de América Latina servió de terreno fértil para las protestas.

El PIB de los países de América Latina y el Caribe exhibe una dinámica regresiva. Si en 2019 el crecimiento del PIB en esos países fue del 1,2\%, uno de los peores índices entre las regiones del mundo, en 2020, según estimaciones, bajaría hasta $9,4 \%[2]$.

Hace tan solo diez años esta región estaba aventajando al resto del mundo en crecimiento económico [3]. La razón de aquello fue la elevada demanda de materias primas por China, 
en pujante desarrollo continuo, lo que había causado el crecimiento brusco de sus precios en el mercado mundial.

Las expectativas de la población de que el auge económico continuase no se cumplieron. Llegó a su fin el boom de materias primas, sus precios cayeron drásticamente en el mercado mundial, lo que agravó bruscamente la situación en la economía de los países latinoamericanos. Muchos iban a enfrentar, tras el desplome de los precios de sus materias primas, el problema del déficit fiscal. Ello condujo a la caída de las inversiones. Como consecuencia del conjunto de dichas razones se vieron congelados los salarios, comenzó a subir la desigualdad social, aumentó la pobreza. Creció también la decepción de la gente ante las políticas de sus gobiernos.

En 2014, a raíz de la caída de precios del petróleo, cuya exportación reportaba más del $90 \%$ de los ingresos en divisas, en Venezuela estalló una grave crisis económica [4]. Brasil sufrió un receso económico de los más prolongados y profundos de su historia. En Argentina se produjo una crisis monetaria y de la deuda, donde el peso se desplomó a un mínimo de récord. Incluso los países de mayor estabilidad, como México, Perú y Colombia experimentaron un estancamiento económico. Los problemas económicos iban aumentando también en Chile.

A medida que el crecimiento económico se desaceleraba y llegaban las olas de escándalos de corrupción, la tensión social y política en el continente iba aumentando.

Según la investigadora norteamericana María Murillo, ello engendró dos tipos de división política en el continente. El primero, las masas populares pobres contra la clase media (Bolivia) y, el segundo, toda la ciudadanía contra las élites (Chile) [5]. 
La pandemia del coronavirus también ha repercutido considerablemente en la situación económica. Esto se ha reflejado en la situación política del continente. Habrá que calcular todavía el daño que la pandemia ha causado en la economía de los países de América Latina. Es evidente que la ruina amenazará en un futuro próximo a la clase media y pequeña en las ramas que se vieron paralizadas en el período de la pandemia. La sucesión de quiebras, la caída de la recaudación de impuestos, el déficit fiscal, la reducción o el congelamiento de los gastos sociales provocarán el crecimiento de la pobreza y el ahondamiento de la desigualdad social.

\section{Síntomas de la inestabilidad política en América Latina}

\section{La debilidad institucional de los sistemas y regímenes políticos de los países de América Latina}

El crecimiento económico y el auge social y cultural relativo que experimentaba el continente hace algunos años no pudieron evitar el debilitamiento institucional de los sistemas políticos [6; 7]. Ello se manifestó, en primer lugar, en la violación de la legitimidad del poder estatal lo que se debe a un conjunto de causas: la tensión política que se iba agudizando a medida que se tornaba claro que la corrupción abarca cada vez más el poder estatal, la ley rige selectivamente con respecto a las personas en dependencia de su estatus y la élite política se hace cada vez menos sujeta al control por parte de la sociedad civil (aquello ocurrió en Bolivia, donde el presidente Evo Morales ignoraba la limitación del plazo de las facultades y se impuso por cuarta vez en las elecciones). Ya antes habían adquirido una notoriedad negativa los comicios de 1988 y de 2006 en México, donde los candidatos oficialistas triunfaron con una ventaja tan mínima 
que dio motivo para cuestionar la legitimidad de Carlos Salinas y Felipe Calderón.

En segundo lugar, en toda la región devino patente la tendencia a la disminución del apoyo a la democracia. La gente ha dejado de creer en las instituciones políticas y los sistemas políticos han resultado incapaces de tomar en cuenta las demandas de las minorías o las necesidades de la mayoría de la población.

En tercer lugar, en una serie de países de América Latina se registra la politización (posiblemente artificial) de una serie de problemas y, sobre esa base, la movilización política de la población. Así, el problema de género adquiere un carácter cada vez más politizado. Sucede la movilización del movimiento feminista en defensa de la igualdad de género, contra la violencia de género y la discriminación. Así como de los representantes de LGBT, en defensa del derecho al matrimonio de personas del mismo sexo.

\section{El crecimiento de la violencia y del narcotráfico e ineficacia de combatirlos}

En América Latina ha sido combatido durante muchos años el crimen organizado y sus brotes, la violencia, el narcotráfico y la corrupción.

En los países del Triángulo Norte de Centroamérica que son El Salvador, Guatemala y Honduras, se generó una situación singularmente grave en cuanto a la violencia, perpetrada por bandas armadas, y a las drogas.

Por supuesto, la situación difiere entre los países productores de narcóticos (Colombia, Perú y Bolivia) y los países de su tránsito, a los que pertenecen los del Triángulo Norte y México. Pero, en la mayoría de los casos resultan estériles los intentos de 
los gobiernos de reprimir la criminalidad con la policía y la fuerza militar.

Hoy día, 17 de los 20 países más violentos del mundo en tasa de asesinatos se encuentran en América Latina y el Caribe. El 95\% de los crímenes cometidos en América Central quedan impunes. [8] La ampliación de las facultades de la policía y el recrudecimiento de los castigos no dan resultados, pero sí llevan a la violación de los derechos humanos.

El incremento de la violencia en los países de América Latina es nutrido por el comercio ilegal y el tráfico de drogas. El comercio de estupefacientes en los países de América Central florece, sobre todo en Colombia. En este país, la producción de cocaína alcanzó un nivel elevado récord llegando a su máximo histórico en 2018 y creciendo un 17\% [8]. La producción y la comercialización de la cocaína han sido un método seguro para financiar a los guerrilleros de las FARC [9].

Los partidarios de la línea dura en la lucha contra el narcotráfico y la violencia vinculada a ello respaldan el endurecimiento de las medidas punitivas y de la militarización del país.

Algunos líderes políticos, al no ver otra salida, apuestan al incremento de los rasgos autoritarios de sus regímenes políticos.

\section{La corrupción y los escándalos ligados con ella}

La región de América Latina sufre el flagelo de la corrupción, estrechamente vinculada a los sindicatos del crimen organizado que se dedican al tránsito y al comercio de drogas. En muchos años se ha formado todo un sistema de sobornos, el que no han logrado erradicar ni las protestas ciudadanas, ni los escándalos políticos en gobiernos de América Latina, ni las 
campañas contra la corrupción de gran envergadura, pero poco eficaces.

En los últimos años, muchos estados latinoamericanos fueron azotados por una ola de escándalos de corrupción. Estos afectaron a Guatemala, Argentina [10], Brasil, Panamá, Colombia, Perú y Haití [11]. La corrupción de gran escala erosiona las instituciones democráticas de la región y conduce al ahondamiento de la crisis política. Esta actividad se ha arraigado sólidamente en los negocios y en las instituciones del Estado, determinando el modo de la interacción del poder económico y político en la América Latina actual. Un ejemplo ilustrativo son los cuatro expresidentes del Perú encarcelados por el delito de corrupción.

Los votantes, al no ver una salida de los problemas de la corrupción, de la violencia y de las drogas, que están estrechamente vinculados, manifiestan su simpatía y dan sus votos o a los candidatos populistas, o a los demócratas prefieren los políticos de "mano dura".

Los pueblos de América Latina esperan de sus gobiernos nuevos esfuerzos para combatir el soborno. Sin embargo, a juicio de Donald Partyka ${ }^{1}$, los resultados de las campañas contra la corrupción en el continente siguen siendo de momento poco eficaces [12].

En la mayoría de los países latinoamericanos, la corrupción no es una enfermedad que ataca algunas instituciones del poder por separado. Ella devino parte inalienable del funcionamiento normal del Estado. En América Latina no solamente algunos políticos o partidos políticos son corruptos, sino que está corrupto el sistema mismo del poder.

\footnotetext{
${ }^{1}$ Donald Partyka, miembro del Consejo de redacción y director creativo de la revista America Quarterly.
} 


\section{Crisis migratoria *}

Los disturbios políticos en Nicaragua y en los países del Triángulo Norte de Centroamérica revisten un carácter violento y se han tornado fuente de un flujo constante de emigración.

Las causas de la migración desde los países del Triángulo Norte son: la falta de posibilidades económicas y la situación penosa de la gente [13], la vulnerabilidad frente a las bandas armadas y a la violencia y la impotencia del gobierno frente a la situación.

Los flujos migratorios en el continente abarcan ya a millones de personas [14]. Desde América Central se busca llegar a EE.UU. a través de México; los refugiados de Venezuela tratan de ingresar a la vecina Colombia y a Brasil, los cesantes de Bolivia buscan trabajo en Chile o Argentina.

La violencia, las drogas, la corrupción y la migración son todo manifestaciones de un problema integral que es la crisis latinoamericana.

\section{Manifestaciones de volatilidad política en América Latina}

\section{El fin del "giro a la izquierda" y la tendencia a una "deriva a la derecha"}

América Latina fue inundada, al comienzo de los años 2000, por la "marea rosa". Aquello coincidió con el auge de la demanda y los precios de las materias primas en los mercados mundiales, del que se beneficiaron los países latinoamericanos proveedores de tales productos. Los líderes izquierdistas no

\footnotetext{
*Véase más: Leónova O. Crisis migratoria en América Latina. Iberoamerica. Moscow, 2019, núm.3, pp. 104-124. 
lograron, desgraciadamente, transformar ese auge de materias primas en un crecimiento económico estable y en el progreso social para todas las capas de la población [15]. Cuando hacia el 2014 llegó el final del boom de las materias primas y se desataron las dificultades económicas, los gobiernos izquierdistas comenzaron a ser relevados por fuerzas de derecha y centroderecha en Brasil, Argentina, Colombia, Uruguay, Ecuador, Paraguay, Perú, el Salvador, Panamá, Guatemala y Honduras [16; 17].

Así, en Argentina en 2015 un exponente de las fuerzas de derecha, Mauricio Macri, pasó a relevar al gobierno peronista. La victoria de Jair Bolsonaro en las presidenciales de Brasil de 2018 puso el punto final al "giro a la izquierda" [18]. Su administración ha sido marcada también por una sucesión de escándalos de corrupción [19]. La política económica de los gobiernos de derecha resultó ineficaz y provocó el desencanto de la ciudadanía. Por ejemplo, las medidas de la austeridad y los préstamos exteriores aplicados por el presidente de Argentina Mauricio Macri llevaron a consecuencias negativas. Como resultado, la deuda total del gobierno federal sumaba, a fines del 2019, el 88\% del PIB, y en mayo de 2020 el país anunció oficialmente la cesación de pagos [20].

A pesar de ello, saltaba a la vista una aparente "deriva a la derecha" del continente. Sin embargo, no todo era tan unívocamente. No obstante, la "deriva a la derecha" en algunos países, las fuerzas de izquierda conservaron e, incluso, fortalecieron sus posiciones [21].

Así, en octubre de 2019, Mauricio Macri fue derrotado en las presidenciales por el candidato de centroizquierda peronista Alberto Fernández. En México, en la República Dominicana, Costa Rica y Panamá llegaron al poder gobiernos de 
centroizquierda. En Bolivia, después de las elecciones generales extraordinarias volvería el Movimiento al Socialismo (MAS). En Venezuela, el Partido Socialista Unido conquistó el control total sobre el parlamento. Las protestas multitudinarias en Chile y Ecuador obligaron a los gobiernos de esos países a atenuar la política económica austera y (por ejemplo, en Chile) pusieron en tela de juicio el modelo económico neoliberal de desarrollo. Andréi Budaiev considera que las protestas sociopolíticas de fines del 2019, en diversos países de América Latina, dieron cuenta de las "fallas sistémicas en la política neoliberal de los gobiernos de derecha" [22, p. 31]. En México, las medidas adoptadas por el presidente López Obrador, respaldadas ampliamente por la población, “están destinadas a enmendar el contenido de las reformas neoliberales que él critica enérgicamente...", apunta Anatoly Borovkov [23, p. 60].

Difícilmente se puede afirmar categóricamente que en América Latina se haya ahogado "el giro a la izquierda". Pero, es evidente la presencia de la "deriva a la derecha". Tal variedad de paisaje político y la ausencia de unidad política llevó a Zbígniev Iwanowski a concluir que el continente hoy en día, tanto en el aspecto político como social, se distingue por una sorprendente diversidad [24, p. 30].

"Pero al mismo tiempo, el "giro a la izquierda" en México tiene una sustancial particularidad que lo diferencia de algunos otros regímenes de los países latinoamericanos. Este régimen puede ser caracterizado de centroizquierdo moderado, con una ausencia total de radicalismo de izquierda", destaca Anatoly Borovkov [25, p. 71]. 


\section{El crecimiento de la actividad política y de la movilización de la población}

En los años 2019 y 2020 estallaron disturbios en una serie de países de América Latina. Como motivo para dichas manifestaciones pudieron servir los escándalos de corrupción, los ajustes en la política económica o los resultados de las elecciones.

Por ejemplo, en Nicaragua, las reformas económicas propuestas por el presidente Daniel Ortega gatillaron las protestas airadas de la oposición.

En Bolivia, las manifestaciones populares estallaron después de que el presidente Evo Morales, uno de los representantes del "giro a la izquierda", intentara hacerse con un cuarto mandato presidencial.

En Colombia, generan el malestar de la ciudadanía los ataques armados incesantes de los bandidos contra los activistas civiles y los líderes de pueblos nativos, centenares de los cuales han sido asesinados.

En Ecuador detonaron las protestas cuando el gobierno redujo los subsidios del combustible y los precios de este subieron el doble.

En Chile, en las manifestaciones participaron más de un millón de personas, un récord histórico para el país [26].

En Brasil, en marzo de 2020, en varias ciudades se llevaron a cabo violentas protestas de representantes de la clase media contra la política presidencial.

Los políticos se empeñan en adoptar algunas medidas, pero, como regla general, todas son de medias tintas y tienen un carácter de compromiso.

La particularidad de estas protestas consiste en que, siendo aparentemente provocadas por los problemas sociales, ellas 
entrañaban también demandas políticas. Por ejemplo, en Bolivia tuvieron lugar manifestaciones multitudinarias de respaldo a Evo Morales, mientras que la oposición demandaba la celebración de elecciones transparentes y la observancia de la legislación. En Chile, ante la demanda de los manifestantes, el gobierno tuvo que acceder a convocar un plebiscito sobre la adopción de una nueva Carta Magna. En Venezuela persiste la polarización entre partidarios y adversarios del gobierno. En Colombia el presidente Iván Duque, bajo la presión de la opinión pública, pactó un acuerdo de paz con la guerrilla de las FARC y se vio obligado a acelerar el proceso de creación de un tribunal para el estudio y el sancionamiento de los crímenes de guerra. En Nicaragua la oposición exigió la renuncia de Daniel Ortega.

La excepción de la regla la representa quizás México, debido a que "...las medidas prácticas del nuevo presidente y de su equipo pueden dar muestra de una nueva variante de gestión de centroizquierda, en medio de un vasto respaldo dentro del país a las medidas populares que han venido adoptando..." [25, p. 72]. Las manifestaciones en México de 2019 y 2020 revistieron un carácter esporádico y tuvieron sus singularidades. En enero de 2019, las protestas se debieron a la crisis del combustible y a la escasez en las gasolineras. En mayo del mismo año, varios millares de manifestantes expresaron su malestar con la política del gobierno en materia de seguridad pública y contra el crimen desenfrenado. En junio de 2020, la capital era recorrida por los manifestantes que expresaban así su solidaridad con las protestas contra el racismo y la brutalidad policial en EE.UU. Y anteriormente, el 8 y 9 de marzo se realizaron en México marchas de mujeres, en las que participaron fundamentalmente 
activistas del movimiento feminista, que se manifestaban contra la violencia y la discriminación de género.

\section{La evolución de los hechos puede llevar, ya sea a la} transformación de los regímenes políticos, ya sea a las represiones políticas.

Un hecho sintomático se vivió en la primavera de 2020 en Brasil, cuando se desencadenaron las protestas contra las instituciones democráticas del país. Durante ellas se llegó a abogar incluso por la vuelta al régimen castrense.

Hay que recordar que el propio Jair Bolsonaro, cuando era un candidato presidencial, en varias ocasiones se expresaba de manera bastante favorable acerca de la dictadura militar del pasado en Brasil. Hoy día, cuando la extrema pobreza y el nivel de criminalidad crecen impetuosamente, cuando las instituciones políticas están del todo desprestigiadas y los brasileños están profundamente desilusionados con los resultados de la política económica, ellos se han abierto a las ideas de políticos tales como Bolsonaro. Aunque, a muchos les inquieta sobremanera que realmente pueda hacer volver al país a una dictadura castrense [27].

Estas tendencias podrían poner en riesgo no solo el futuro de un país concreto, sino el futuro de la democracia en toda América Latina. Sin embargo, paralelamente con la probabilidad del reforzamiento de las tendencias autoritarias, existe una tendencia a la democratización en algunos países. En estos, las elecciones transcurren a tiempo, de conformidad con la Constitución y sobre la base de candidaturas alternativas y de la competencia; los partidos opositores no están prohibidos y trabajan activamente los parlamentos. En Chile se llevó a cabo un plebiscito y fue aprobada la decisión sobre la reforma constitucional, gracias a lo que se perfiló una salida de la crisis. 
En Bolivia se celebraron las elecciones extraordinarias, en Cuba fueron separadas las facultades del presidente y del jefe del gabinete y fue limitado el plazo de sus facultades, etc.

\section{Conclusiones}

Podemos concluir que la situación política en el continente ha estado condicionada, de una parte, por la crisis que atraviesa hoy en día la globalización $\mathrm{y}$, de otra parte, por los problemas endémicos de América Latina. En los países del continente se ha generado toda una serie de factores desfavorables, cuyo efecto sumario ha adquirido un carácter sistémico integral [28]. Los esfuerzos para combatirlos han sido estériles, pero la necesidad de resolver dichos problemas estimula la búsqueda de una salida que algunas élites políticas podrían ver en el repliegue de la democracia y el fortalecimiento de los regímenes autoritarios.

Ello podría ser el inicio de un ciclo que había conducido a su tiempo a regímenes represivos y a dictaduras militares en América Latina. Hace medio siglo, esos mismos mecanismos habían llevado al continente al caos y a la división.

Sin embargo, la presente situación no es tan solo una prueba severa, sino que podría llegar a ser un momento crucial y abrir el camino al futuro. ¿A dónde $\mathrm{y}$ a qué futuro? Este puede ser distinto.

Esta situación presenta también un momento positivo: la unanimidad política de los jefes de Estado de las fuerzas de derecha amplía las posibilidades de la colaboración de los países de la región.

¿Cuáles son las perspectivas de una salida para América Latina de la situación que se ha formado? 
En primer lugar, para que América Latina vuelva a las tasas elevadas de crecimiento económico es indispensable un programa sincronizado de reformas económicas que rebase el marco de las promesas estándares de los populistas, pero concentrada en dos o tres lineamientos prioritarios.

En segundo lugar, las posiciones políticas afines permiten a los gobiernos de los países del continente sumar esfuerzos y trazar medidas sincronizadas en la lucha contra la corrupción, la violencia y las drogas.

En tercer lugar, persiste la perspectiva de la ulterior integración regional. La situación de los procesos integracionistas en el continente es bastante contradictoria. Por una parte, se observa la desaceleración de los procesos integracionistas: en MERCOSUR se han agudizado las divergencias entre Argentina y Brasil; UNASUR, debido a la imposibilidad de elaborar una postura única con respecto a Venezuela, cerró su sede y, en los hechos, ha dejado de existir. Brasil abandonó la CELAC, una comunidad que sin éste y a raíz de las disensiones políticas se ha transformado en una suerte de club de discusión.

De otra parte, la integración regional recibe estímulos para el desarrollo gracias al consenso de ideas de las fuerzas de derecha que han llegado al poder en la mayoría de los países latinoamericanos. Las relaciones mutuas entre los jefes de Estado de América Latina, que tienen criterios políticos y una visión común de las perspectivas del desarrollo de la economía pueden resolver numerosos problemas de la integración regional. Por ejemplo, Zbígniev Iwanowski considera que el predominio de los gobiernos de derecha (a excepción de México) crea en la Alianza del Pacífico la posibilidad para su acercamiento con MERCOSUR, para la incorporación de esos 
dos bloques subregionales en la Asociación Transatlántica y la integración en la economía global. Se ha creado el supranacional y políticamente bastante homogéneo Grupo de Lima, en el que, además de los países de América del Sur y Central entraron Canadá y Santa Lucía [17].

En cuarto lugar, como consecuencia de la difícil situación económica y política, en el continente se podría suceder un viraje en la política exterior de los países latinoamericanos. Ha comenzado ya a notarse un cierto debilitamiento de la influencia de EE.UU. en América Latina, aunque sus posiciones económicas en el continente siguen siendo sólidas y existe la probabilidad de que la administración de Joe Biden vaya a prestar una atención mayor a esta dirección. Sin embargo, las élites políticas están estudiando ya la posibilidad del ahondamiento de sus vínculos con China, confiando en su respaldo económico sin presiones políticas de su parte.

América Latina se encuentra hoy día en una encrucijada. El continente está en búsqueda de nuevas ideas, nuevos modelos económicos y sociales. El precio que los países de América Latina deberán pagar por la crisis o, por la senda al futuro dependerá, próximamente, de la opción y de las decisiones de la actual élite política.

\section{Bibliografía References Библиография}

1. Globalization, Inclusion and Sustainability in a Global Centure. G 7 International Forum. Rome, May $8^{\text {th }}-9^{\text {th }}$ 2017. Rome, Italy: Eurilink University Press, 2017, $187 \mathrm{p}$.

2. Latest World Economic Outlook Growth Projections. International Monetary Fund. Available at: https://www.imf.org/en/Publications/WEO/Issues/2020/06/24/WEOUpdateJu ne2020 (accessed 16.08.2020).

3. Armendáriz B. and Larraín F. The Economics of Contemporary Latin America. The MIT Press, 2017, 464 p. 
4. Alekséenko O. A., Pyatakov A. N. Venezuela: prueba por la crisis. Iberoamérica. Moscow, 2019, núm. 2, pp. 57-83.

5. Murillo M.V. Why Is South America In Turmoil? Americas Quarterly. November $19,2019 . \quad$ Available at: https://www.americasquarterly.org/content/why-south-america-turmoiloverview?utm_source=AQ\%27s+Week+in+Review\&utm_campaign $=5 \mathrm{fb} 067$ 45da-

EMAIL_CAMPAIGN_2019_08_17_06_51_COPY_01\&utm_medium=email \&utm_term=0_6a33e16b5d-5fb06745da-26145201 (accessed 04.12.2019).

6. Larraín F. Missed Opportunities: The Economic History of Latin America. October 5, 2017. International Monetary Fund. Available at: https://www.imf.org/en/News/Articles/2017/10/05/NA100517-Missed-

Opportunities-The-Economic-History-of-Latin-America (accessed 20.08.202).

7. Crisis políticas y sociales ponen a prueba la gobernabilidad en América Latina. Revista América Economía, 31.12.2019. Available at: https://www.americaeconomia.com/politica-sociedad/politica/crisis-politicasy-sociales-ponen-prueba-la-gobernabilidad-en-america 21.01.2020).

8. Charles M. A Split Emerges in Latin America Over How to Deal With Rising Violence. World Politics Review. Tuesday, Jan. 8, 2019. Available at: https://www.worldpoliticsreview.com/articles/27122/a-split-emerges-in-latinamerica-over-how-to-deal-with-rising-violence (accessed 11.01.2019).

9. Charles M. Why Colombia's Dissident FARC Rebels Are Taking Up Arms Again. World Politic Review. September 4, 2019. Available at: https://www.worldpoliticsreview.com/articles/28163/why-colombia-sdissident-farc-rebels-are-taking-up-arms-again (accessed 20.08.2020).

10. Morayta I. A. Relaciones entre élites: "Captura política" y corrupcion. El caso de Argentina (2015-2019). Iberoamérica. Moscow, 2020, núm. 4 pp. 145-166.

11. Miller, B and Uriegas, F. Latin America's Biggest Corruption Cases: A Retrospective. Americas Quarterly. July 22, 2019. Available at: https://www.americasquarterly.org/content/decades-most-iconic-corruptioncases (accessed 20.08.2020).

12. Partyka D. Is Latin America's Corruption Crackdown Falling Apart? Americas Quarterly. July 10, 2019. Available at: https://www.americasquarterly.org/content/corruption-issue-leader-english (accessed 20.08.2020).

13. Vershínina I.M. Honduras: problemas socioeconómicos como causa de la emigración. Iberoamérica. Moscow, 2019, núm.1, pp. 88-105. 
14. Selee Andrew. Latin America's Migration Lesson for the World. America Quarterly. March 18, 2019. Available at: https://www.americasquarterly.org/content/latin-americas-migration-lessonworld (accessed: 31.08.2020).

15. Razumovskiy D. V. Consecuencias económicas del gobierno de los regímenes de izquierda en Argentina y Brasil. Iberoamérica. Moscow, 2018, núm.1, pp. 50-78.

16. O'Neil Sh. K. Latin America's Right Turn Could Draw Its Economies Closer. The ascent of leaders who favor free trade opens space for real integration. Center of Foreign Relations. February 26, 2019. Available at: https://www.cfr.org/blog/latin-americas-right-turn-could-draw-its-economiescloser (accessed 18.07.2020).

17. Ивановский 3.В. Латинская Америка в новом тысячелетии: социальная панорама и динамика политических процессов (окончание). Латинская Америка. M., 2019, №8, c. 6-22. [Iwanowski Z.W. Latinskaya Amerika $\mathrm{v}$ novom tysyacheletii: social'naya panorama i dinamika politicheskikh processov (okonchanie) [Latin America in the New Millennium: a social panorama and the dynamics of political processes (ending)] Latinskaya Amerika. Moscow, 2019, num. 7, pp. 30-39 (In Russ.)].

18. Ókuneva L. S. Brasil consolida su rumbo a la derecha. Iberoamérica. Moscow, 2019, núm.4, pp. 44-62.

19. Da Silva D. and Abramovay P. Target Corruption's Roots, Not Just Its Leaves. Americas Quarterly. July, 2019. Available at: https://www.americasquarterly.org/content/tackling-corruption-roots (accessed 20.08.2020).

20. Clavijo J. Once the 'Country of the Future,' Can Argentina Free Itself From Its Past? World Politic Review. June 30, 2020. Available at: https://www.worldpoliticsreview.com/articles/28879/in-the-wake-of-defaultargentina-struggles-to-break-from-its-past (accessed 20.08.2020).

21. Ивановский 3.В. Политическая поляризация в Латинской Америке. Итоги политического цикла. Свободная мыслль. М., 2019, №1, c. 149-168 [Iwanowski Z.W. Politicheskaya polyarizatsiya v Latinskoy Amerike. Itogi politicheskogo tsikla [Political polarization in Latin America. Results of the political cycle]. Svobodnaya mysl'. Moscow, 2019, num. 1, pp. 149-168 (In Russ.)].

22. Будаев А. В. «Латиноамериканская весна - 2019». Кризис неолиберальной модели развития. Латинская Америка. М., 2020, № 6, c. 31-41 [Budaev A.V. "Latinoamerikanskaya vesna - 2019». Krizis neoliberal'noy modeli razvitiya ["Latin American Spring-2019". The crisis of the neoliberal development model]. Latinskaya Amerika. Moscow, 2020, num. 6, pp. 31-41 (In Russ.)]. 
23. Боровков А. Н. Россия - Мексика: исторические параллели в отношениях двух стран. К 130-летию дипломатических отношений. Латинская Америка. М., 2020, №11, с. 52-66. [Borovkov A.N. Rossiya Meksika: istoricheskie paralleli v otnosheniyah dvukh stran. K 130-letiyu diplomaticheskih otnosheniy [Russia - Mexico: historical parallels in relations between the two countries. On the 130th anniversary of diplomatic relations]. Latinskaya Amerika. Moscow, 2020, num. 11, pp. 52-66 (In Russ.)].

24. Ивановский 3. В. Латинская Америка в новом тысячелетии: социальная панорама и динамика политических процессов (часть 1). Латинская Америка. M., 2019, №7, с. 30-39 [Iwanowski Z.W. Latinskaya Amerika $\mathrm{v}$ novom tysyacheletii: social'naya panorama i dinamika politicheskih processov (chast' 1) [Latin America in the New Millennium: a social panorama and the dynamics of political processes (part 1)]. Latinskaya Amerika, Moscow,2019, num. 7, pp. 30-39 (In Russ.)].

25. Боровков А. Н. Мексика на новом историческом повороте. Латинская Америка. М., 2019, №7, с. 61-75 [Borovkov A. N. Meksika na novom istoricheskom povorote [Mexico is on a new historical turn]. Latinskaya Amerika. Moscow, 2019, num. 7, pp. 61-75 (In Russ.)].

26. Cheatham A. What's Behind the Chile Protests? Center of Foreign Relations. November 1, 2019. Available at: https://www.cfr.org/inbrief/whats-behind-chileprotests?utm_source=dailybrief\&utm_medium=email\&utm_campaign=Daily Brief2020Mar20\&utm_term=DailyNewsBrief (accessed 20.08.2020).

27. Ghitis F. Is a Far-Right Wave Building in Latin America, Beginning in Brazil? World Politic Review. October 11, 2018. Available at: https://www.worldpoliticsreview.com/articles/26346/is-a-far-right-wavebuilding-in-latin-america-beginning-in-brazil (accessed 18.08.2020).

28. Яковлева Н.М., Яковлев П.П. Три волны системного кризиса в Латинской Америке. Латинская Америка. М., 2020, №10, с. 6-20 [Yakovleva N. M., Yakovlev P. P. Tri volny sistemnogo krizisa v Latinskoj Amerike [Three waves of systemic crisis in Latin America]. Latinskaya Amerika. Moscow, 2020, num. 10, pp. 6-20 (In Russ.)]. 